Special Issue of the 6th International Congress \& Exhibition (APMAS2016), Maslak, Istanbul, Turkey, June 1-3, 2016

\title{
Optoelectronic, Elastic and Thermal Properties of Cubic Perovskite-Type $\mathrm{SrThO}_{3}$
}

\author{
Y. Benaissa Cherif ${ }^{a, *}$, M. Rounighia ${ }^{a}$, A. Zaoui $^{b}$ And A. BoukortT ${ }^{a}$ \\ ${ }^{a}$ Elaboration Characterization Physico Mechanics of Materials and Metalurgical Laboratory (ECP3M), \\ Faculty of Sciences and Technology Abdelhamid Ibn Badis, Mostaganem University, 27000, Algeria \\ ${ }^{b}$ Laboratoire de Physique Computationnelle des Matériaux, Université Djillali Liabés de Sidi Bel-Abbès, \\ Sidi Bel-Abbès 22000, Algeria
}

\begin{abstract}
The electronic structure, elastic, thermal and optical properties of the cubic strontium thorate $\mathrm{SrThO}_{3}$ are calculated using the full-potential linearized augmented plane wave method (FP-LAPW) based on the density functional theory with generalized gradient approximations GGA and local density approximation LDA. The modified Becke-Johnson potential (mBJ) is applied in electronic structure for calculating the energy gap. The obtained results are the Young modulus, shear modulus, the Poisson ratio, isotropic shear modulus, longitudinal, transverse and average sound velocities, the Zener anisotropy factor, the Kleinman parameter and the Debye temperature of the systems. All results are discussed and compared with the available experimental data.
\end{abstract}

DOI: 10.12693/APhysPolA.131.406

PACS/topics: 78.20.Ci, 65.40.-b, 65.40.--b

\section{Introduction}

Broadly speaking, perovskites are important materials for optoelectronics field, where many studies of their physical properties are part of scientific research today. They reveal much more important properties from theoretical and experimental point of view, such as ferroelectricity, superconductivity, optical and magnetic properties $[1,2]$.

The first synthesis of $\mathrm{SrThO}_{3}$ by conventional solid route was reported in 1947 by Marie-Szabo [3]. Recently, Subasri et al. [4] have noted the limited thorium oxide solubility in $\mathrm{SrO}$ and that the formation of the pure ternary phase is not achieved. On the other hand, the samples of $\mathrm{SrThO}_{3}$ are prepared correctly by a sol-gel technique followed by the combustion of gel $[5,6]$.

The properties of perovskites, such as strontium thorate $\mathrm{SrThO}_{3}$, have attracted a lot of interest over the last decade [4-7]. The values of the Gibbs energy of $\mathrm{SrThO}_{3}$ show that this compound is meta-stable with respect to its constituent oxides ( $\mathrm{SrO}$ and thorium), and therefore it is difficult to eliminate the synthesis of strontium thorate. $\mathrm{SrThO}_{3}$ is reported to be prepared by a conventional solid state route [8, 9], Purohit et al. [7] have studied the synthesis of nano-crystalline powders of $\mathrm{SrThO}_{3}$ by a gel combustion route. To date we have the structural data [3-7]. The coefficients of linear thermal expansion [7] were also reported. Shein et al. have calculated the electronic and elastic properties of $\mathrm{SrThO}_{3}$ in the cubic phase [10], using the FPLAPW method within GGA approximation. Though there is some experimental and theoretical work on the compound, however any detailed data concerning the optical properties of $\mathrm{SrThO}_{3}$ is probably not available in literature.

*corresponding author; e-mail: youcher2003@yahoo.fr
In this work, we will contribute to the study of the structural, elastic, thermal, electronic and optical properties of $\mathrm{SrThO}_{3}$ using the full potential linearized augmented plane wave (FP-LAPW) method in the densityfunctional theory (DFT) framework within GGA, LDA and $\mathrm{mBJ}$ approximation using the WIEN2K code.

\section{Computational method}

The first-principles calculations are performed using the full-potential augmented plane wave (FP-LAPW) method as implemented in WIEN2K code [11]. The exchange-correlation potential was calculated within the local density approximation (LDA), developed by Ceperley and Alder and parameterized by Perdew and Zunger $[12,13]$, as well as the generalized gradient approximation (GGA) of Perdew, Burke and Ernzerhof [14]. We have used the Tran and Blaha modified BeckeJohnson potential $[15,16](\mathrm{mBJ})$ for calculating the electronic properties of the material. The $\mathrm{mBJ}$ functional cannot be used for total energy calculations but yields improved band gaps in a wide variety of materials [15-17]. In this work, we have introduced 2300 plane waves in the structure of the the cubic perovskite $\mathrm{SrThO}_{3}$. A satisfactory degree of convergence is achieved by considering a number of basic functions FP-LAPW $R_{\mathrm{MT}} K_{\max }=7$, where $R_{\mathrm{MT}}$ is the average radius of the Muffin-Tin spheres and $K_{\max }$ is the maximum value of the wave vector $K=k+G$.

The elastic coefficients were determined from firstprinciples calculations by applying a set of given homogeneous deformations with a finite value and calculating the resulting stress with respect to optimizing the internal atomic degrees of freedom [18]. A cubic crystal has three independent elastic constants, namely $C_{11}, C_{12}$, and $C_{44}$. One strain pattern, with non-zero first and fourth components, gives stresses related to all three independent elastic coefficients for the cubic system. Three positive 
and three negative amplitudes were used for each strain component with the maximum value of $0.5 \%$, and then the elastic stiffness coefficients were determined from a linear fit of the calculated stress as a function of strain.

The study of thermal effects was done within the quasiharmonic Debye model implemented in the Gibbs program [19]. For a solid described by an energy-volume $(E-V)$ relationship in the static approximations, the Gibbs program allows us to evaluate the Debye temperature, to obtain the Gibbs free energy $G(V ; P, T)$ and to minimize $G$ for deriving the thermal equation of state $V(P, T)$. Other macroscopic properties related to $\mathrm{P}$ and $\mathrm{T}$ can be also derived by using standard thermodynamic relations $[19,20]$.

\section{Results and discussion}

\subsection{Structural properties}

The calculated structural properties of $\mathrm{SrThO}_{3}$, using the FP-LAPW method within both the LDA and the GGA approximations, are quoted in Table I along with the available experimental and theoretical data. It is evident from the table that our calculated lattice parameter is in good agreement to the experimental [21] and other theoretical results [22].The calculated lattice parameters $(a, b$ and $c$ ) using the LDA and GGA approximations are $4.49 \AA$ and $4.52 \AA$ respectively, which show a good agreement with experimental values. Consequently, the GGA approximation was found to be very successful when applied to systems of perovskite $\mathrm{SrThO}_{3}$ (see Fig. 1).

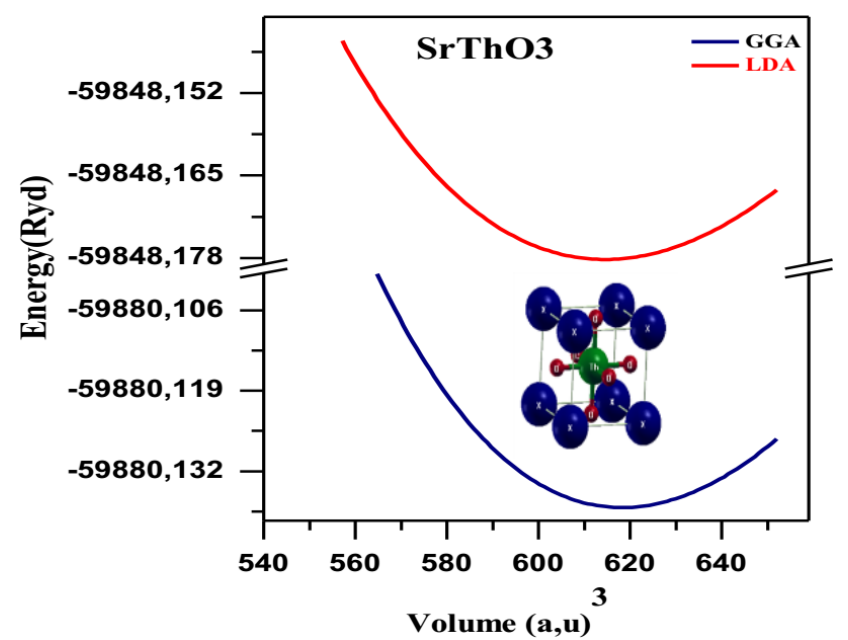

Fig. 1. The variation of the total energy as a function of the volume of $\mathrm{SrThO}_{3}$.

The lattice constants of the perovskites are also calculated by the ionic radius method, using the following relation [23]:

$$
a_{0}=\alpha+\beta\left(r_{\mathrm{Sr}}+r_{\mathrm{O}}\right)+\gamma\left(r_{\mathrm{Th}}+r_{\mathrm{O}}\right),
$$

where $\alpha=0.06714, \beta=0.4905, \gamma=1.2991$ [24], $r_{\mathrm{Sr}}$ is the ionic radius of $\operatorname{Sr}(1.44 \AA)$ and $r_{\mathrm{Th}}$ is the ionic radius of $\mathrm{Th}(0.94 \AA)$, while $\mathrm{r}_{\mathrm{O}}$ is the ionic radius of $\mathrm{O}(1.35 \AA)$.
It is clear from the table that our lattice constants calculated by ionic radius method are $4.49 \AA$ for $\mathrm{SrThO}_{3}$, is in close agreement (less than $1 \%$ ) with the experimental results [20-21]. Critical radius plays an important role in the activation energy of oxygen migration, ion conductivity and it also provides a guideline for doping selection. The critical radii for the compound are calculated by using the following mathematical formula [25, 26]:

$$
\begin{aligned}
& r_{\mathrm{c}}=\frac{-r_{\mathrm{A}}^{2}+\frac{3}{4} a^{2}-\sqrt{2} a r_{\mathrm{B}}+r_{\mathrm{B}}^{2}}{2 r_{\mathrm{A}}+\sqrt{2} a-2 r_{\mathrm{B}}}, \\
& a=2\left(r_{\mathrm{B}}+r_{\mathrm{O}}\right) t<1, \\
& t=\frac{r_{\mathrm{A}}+r_{\mathrm{O}}}{\sqrt{2}\left(r_{\mathrm{A}}+r_{\mathrm{B}}\right)} .
\end{aligned}
$$

The values of the bulk modulus are $120.17 \mathrm{GPa}$ (127.59 GPa), using GGA (LDA) approach and with a pressure derivative which is equal to 4.062 .

TABLE I

Calculated, analytic and experimental values of lattice constants, volume, bulk modulus, pressure derivative of bulk modulus, band gap $E_{g, \Gamma-\Gamma}, E_{g}(\mathrm{mBj})$ and critical radii $r_{\mathrm{c}}$, tolerance factors, elastic constants, Zener anisotropy factor $A$, Poisson's ratio $v$, Kleinman parameter $\zeta$, Young's modulus $E$, and shear modulus $C^{\prime}$ of $\mathrm{SrThO}_{3}$.

\begin{tabular}{c|c|c|c|c|c}
\hline \hline Param. & Experim. & $\begin{array}{c}\text { LDA } \\
\text { GGA }\end{array}$ & $\begin{array}{c}\text { Present } \\
\text { work }\end{array}$ & $\begin{array}{c}\text { Other } \\
\text { work }\end{array}$ & $\begin{array}{c}\text { Analytical } \\
\text { work }\end{array}$ \\
\hline$a[\AA]$ & $\begin{array}{c}4.5426^{a} \\
4.42^{b}\end{array}$ & 4.49 & 4.53 & $4.43^{c}$ & $4.316^{d}$ \\
vol. $\left[\AA^{3}\right]$ & & 611.8017 & 627.1834 & & \\
$B[\mathrm{Gpa}]$ & & 127.5928 & 120.1704 & & \\
$B^{\prime}$ & & 4.1011 & 4.0620 & & \\
$E_{g, \Gamma-\Gamma}$ & & 1.912 & 2.058 & $3.00^{a}$ & \\
$E_{g}(\mathrm{mBj})$ & & 4.202 & 4.230 & & \\
$r_{c}$ & & & & & 0.653 \\
$t$ & & & & 0.780 & 0.8614 \\
$\mathrm{C}_{11}$ & & 141.98 & 209.64 & $197.3^{a}$ & \\
$\mathrm{C}_{12}$ & & 95.72 & 72.33 & $67.10^{a}$ & \\
$\mathrm{C}_{44}$ & & 32.59 & 32.18 & $31.90^{a}$ & \\
$A$ & & 1.40 & 0.47 & $0.49^{a}$ & \\
$v$ & & 0.551 & 0.476 & $0.467^{a}$ & \\
$\zeta\left[\mathrm{kg} / \mathrm{m}^{3}\right]$ & & 0.12 & 0.13 & $0.229^{a}$ & \\
$E[\mathrm{GPa}]$ & & 78.54 & 116.98 & $113.31^{a}$ & \\
$C^{\prime}[\mathrm{GPa}]$ & & 23.13 & 68.65 & $65.10^{a}$ & \\
\hline
\end{tabular}

a:[21]; b:[20]; c:[3]; d:[25].

\subsection{Elastic properties}

For cubic system, there are three independent elastic constants $\left(\mathrm{C}_{11}, \mathrm{C}_{12}\right.$ and $\left.\mathrm{C}_{44}\right)$. Each of them represents the directional mechanical responses of the crystal for different directions of applied forces [27]. In this work, we have used the IRelast method developed by J.Morteza and implemented in the WIEN2K package [11]. The elastic constants $\left(\mathrm{C}_{i j}\right)$ for cubic $\mathrm{SrThO}_{3}$ calculated using the FP-LAPW method within both LDA and GGA approximations, are summarized in Table I. 
Under pressure $P$, the calculated elastic constants are positive and satisfy the generalized elastic stability criteria [28, 29]:

$$
\begin{aligned}
& \frac{1}{2}\left(\mathrm{C}_{11}-\mathrm{C}_{12}-2 P\right)>0, \\
& \frac{1}{3}\left(\mathrm{C}_{11}+\mathrm{C}_{12}+P\right)>0, \\
& \left(\mathrm{C}_{44}-P\right)>0, \quad \text { see Table } \mathrm{I} .
\end{aligned}
$$

The bulk modulus $B$ should also satisfy the criterion: $\mathrm{C}_{12}<B<\mathrm{C}_{11}$.

The bulk modulus $B$, calculated by the formula

$$
B=\frac{1}{3}\left(\mathrm{C}_{11}+2 \mathrm{C}_{12}\right) \text {, }
$$

is similar to those obtained by LDA and GGA approximation.

The most interesting elastic constants (listed in Table II), the anisotropy factor $A$, Poisson's ratio $v$, Young's modulus $E$, isotropic shear modulus $G$, Kleinman parameter $\zeta$ and shear modulus $C^{\prime}$ can be calculated using the following relations [30]:

$$
\begin{aligned}
& A=\frac{2 \mathrm{C}_{44}}{\mathrm{C}_{11}-\mathrm{C}_{12}}, \quad v=\frac{3 B-2 G}{2(2 B+G)}, \\
& E=\frac{9 G B}{G+3 B} \quad \text { and } \quad G=\frac{G_{\mathrm{V}}+G_{\mathrm{R}}}{2} .
\end{aligned}
$$

Here $G_{\mathrm{V}}$ is Voigt's shear modulus, corresponding to the upper bound of $G$ values and $G_{\mathrm{R}}$ is Reuss's shear modulus, corresponding to the lower bound of $G$ values. They can be expressed as:

$$
\begin{aligned}
& G_{\mathrm{V}}=\frac{\mathrm{C}_{11}-\mathrm{C}_{12}+3 \mathrm{C}_{44}}{5}, \\
& \frac{5}{G_{\mathrm{R}}}=\frac{4}{\left(\mathrm{C}_{11}-\mathrm{C}_{12}\right)}+\frac{3}{\mathrm{C}_{44}}, \\
& \zeta=\frac{\mathrm{C}_{11}+8 \mathrm{C}_{12}}{7 \mathrm{C}_{11}+2 \mathrm{C}_{12}} .
\end{aligned}
$$

And shear modulus [31] is given by:

$$
C^{\prime}=\frac{\mathrm{C}_{11}-\mathrm{C}_{12}}{2} \text {. }
$$

The Kleinman parameter $\zeta$ describes the relative positions of cation and anion sub lattices under volume conserving strain distortions for which positions are not fixed by symmetry.

The anisotropy factor $A$ is equal to one for an isotropic material, while any value smaller or larger than one indicates anisotropy. We obtain that the value of the anisotropy factor $A$ is 0.85 in the LDA (0.47 in the GGA). This indicates that our compound is anisotropic. Young's modulus $E$ is a good indicator about the stiffness of the material. When it is higher for a given material, the material is stiffer. Poisson's ratio $v$ provides more information for dealing with the characteristic of the bonding forces than does any of the other elastic properties. The value of the Poisson ratio $v$ for covalent materials is small $(v<0.1)$, whereas for ionic materials a typical value of $v$ is 0.25 [32]. In our calculations $v$ is 0.551 for
LDA and 0.476 for GGA. Hence, a higher ionic contribution in an inter-atomic bonding for this compound should be assumed. The shear modulus $G$ represents the resistance to plastic deformation, while the bulk modulus $B$ represents the resistance to fracture. We know that there is a criterion for $B / G$ ratio which separates the ductility and brittleness of materials. According to Pugh's criteria [33], the critical value is 1.75 , if $B / G>1.75$ the material is ductile, otherwise it is brittle. For the $\mathrm{SrThO}_{3}$, the $B / G$ ratio within LDA is 3.91 and 2.69 within the GGA. Thus according to Pugh's criteria, our material is ductile. Besides, for covalent and ionic materials, the typical relations between bulk and shear modulus are: $G=1.1 B$ and $G=0.6 B$, respectively. For $\mathrm{SrThO}_{3}$ the calculated values of $\mathrm{G} / \mathrm{B}$ are 0.31 within $\mathrm{LDA}$ and 0.37 within the GGA, indicating that the ionic bonding is suitable, which is upper than 1.75. Thus $\mathrm{SrThO}_{3}$ is naturally ductile. The Kleinman parameter $\zeta$ quantifies internal strain and thus indicates the relative ease of bond bending against bond stretching. It also implies resistance against bond bending or bond angle distortion. In a system, minimizing of bond bending leads to $\zeta=0$ and minimizing of bond stretching leads to $\zeta=1$. In the present study, the parameter $\zeta$ is found to be 0.12 within LDA and 0.13 within GGA. It is clear that $\mathrm{SrThO}_{3}$ shows more resistance to bond bending and bond angle distortion among the materials. The Debye temperature is a fundamental physical parameter which is closely related to many physical properties, such as specific heat and melting temperature. At low temperatures, the vibrational excitations arise solely from acoustic vibrations. Hence, at low temperatures the Debye temperature calculated from elastic constants is the same as that determined from specific heat measurements. The Debye temperature $\theta_{\mathrm{D}}$ is calculated from the elastic constants data using the average sound velocity $\vartheta_{\mathrm{m}}$, by the following common relation [34]:

$$
\theta_{\mathrm{D}}=\frac{h}{k}\left[\frac{3 n}{4 \pi}\left(\frac{N_{\mathrm{A}} \rho}{M}\right)\right]^{\frac{1}{3}} \vartheta_{\mathrm{m}},
$$

where $h$ is Planck's constant, $k$ is Boltzmann's constant, $\mathrm{N}_{\mathrm{A}}$ is Avogadro's number, $n$ is the number of atoms per formula unit, $M$ the molecular mass per formula unit, $\rho=M / V$ is the density, and $\vartheta_{m}$ is given [35] as:

$$
\vartheta_{m}=\left[\frac{1}{3}\left(\frac{2}{\vartheta_{\mathrm{t}}^{3}}+\frac{1}{\vartheta_{\mathrm{l}}^{2}}\right)\right]^{\frac{1}{3}} .
$$

Here $\vartheta_{1}$ and $\vartheta_{\mathrm{t}}$ are the longitudinal and transverse elastic wave velocities, respectively, which are obtained from Napier's equations [36]:

$$
\vartheta_{1}=\left(\frac{3 B+4 G}{3 \rho}\right)^{\frac{1}{2}}, \quad \vartheta_{t}=\left(\frac{G}{\rho}\right)^{\frac{1}{2}} .
$$

The calculated Debye temperature and sound velocities, as well as the density of the $\mathrm{SrThO}_{3}$ compound are given in Table II. Our calculated $\vartheta_{\mathrm{l}}, \vartheta_{\mathrm{t}}$ and $\theta_{\mathrm{D}}$ values are $4761.8 \mathrm{~m} / \mathrm{s}, 2079.2 \mathrm{~m} / \mathrm{s}$ and $263.9 \mathrm{~K}$, respectively, within LDA, are to be compared with the values of $\vartheta_{\mathrm{l}}=5148.1 \mathrm{~m} / \mathrm{s}, \vartheta_{\mathrm{t}}=2564.8 \mathrm{~m} / \mathrm{s}$ and $\theta_{D}=324.9 \mathrm{~K}$ within GGA. 
TABLE II

Calculated values of the isotropic shear modulus $G$, longitudinal sound velocity $\vartheta_{1}$, transverse sound velocity $\vartheta_{\mathrm{t}}$, average sound velocity $\vartheta_{\mathrm{m}}$ and Debye temperature $\theta_{\mathrm{D}}$.

\begin{tabular}{c|c|c|c|c|c|c}
\hline \hline & $\mathrm{SrThO}_{3}$ & $\begin{array}{c}\rho \\
{\left[\mathrm{kg} / \mathrm{m}^{3}\right]}\end{array}$ & $\begin{array}{c}\vartheta_{\mathrm{l}} \\
{[\mathrm{m} / \mathrm{s}]}\end{array}$ & $\begin{array}{c}\vartheta_{\mathrm{t}} \\
{[\mathrm{m} / \mathrm{s}]}\end{array}$ & $\begin{array}{c}\vartheta_{\mathrm{m}} \\
{[\mathrm{m} / \mathrm{s}]}\end{array}$ & $\begin{array}{c}\theta_{\mathrm{D}} \\
{[\mathrm{K}]}\end{array}$ \\
\hline Our & ${ }^{a} \mathrm{LDA}$ & 6571.8 & 4761.9 & 2079.2 & 2347.9 & 263.93 \\
work & ${ }^{a} \mathrm{GGA}$ & 6660.5 & 5148.1 & 2564.8 & 2877.9 & 324.95 \\
\hline
\end{tabular}

${ }^{a}$ :IRelast method

\subsection{Thermodynamic properties}

The thermal properties of $\mathrm{SrThO}_{3}$ are determined in the temperature range from 0 to $1200 \mathrm{~K}$, where we have applied the quasi-harmonic Debye approximation. The pressure effect is studied in the range from 0 to $15 \mathrm{GPa}$. In order to determine the structural parameters at $P=0$ and $T=0$, we have fitted with a numerical EOS the total energy versus primitive cell volume, and then derive the macroscopic properties as function of $P$ and $T$ from standard thermodynamic relations.

In Fig. 2, we have plotted the temperature effects on the lattice parameters of $\mathrm{SrThO}_{3}$ at several pressures. The lattice constant increases with increasing temperature at a given pressure. The effect of increasing temperature on the lattice parameter is just the same as that of the decreasing pressure.

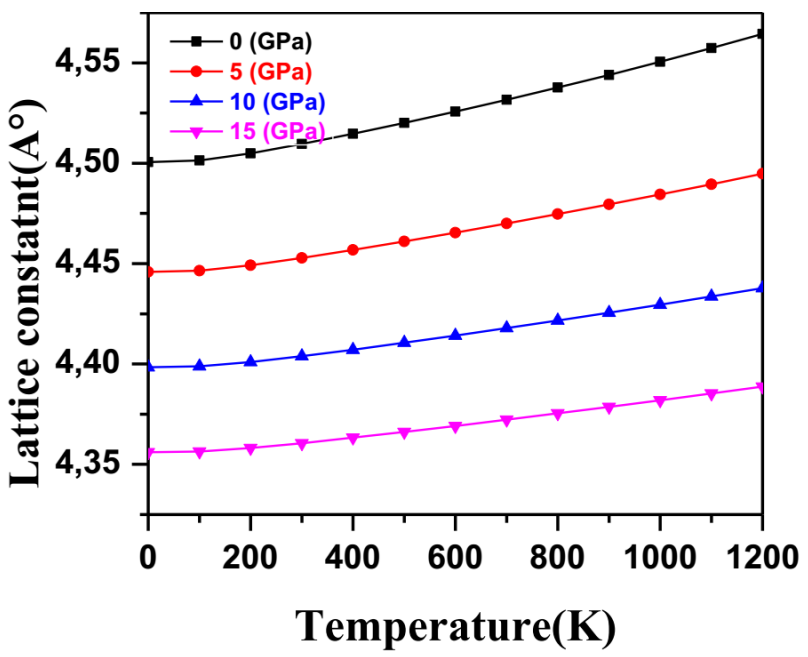

Fig. 2. Variation of the lattice constant as a function of temperature at pressure of $0,5,10$ and $15 \mathrm{GPa}$ for $\mathrm{SrThO}_{3}$.

On the other hand, it is noted from Fig. 3 that the bulk modulus is nearly constant from 0 to $100 \mathrm{~K}$ and decreases linearly with increasing temperature from $T>200 \mathrm{~K}$. The effect of increasing pressure on the material is the same as the that of the decreasing temperature.

We present in Fig. 4 the variation of the thermal expansion coefficient $\alpha$ as function of temperature and pressure. It is shown that, at a given pressure, $\alpha$ increases with the

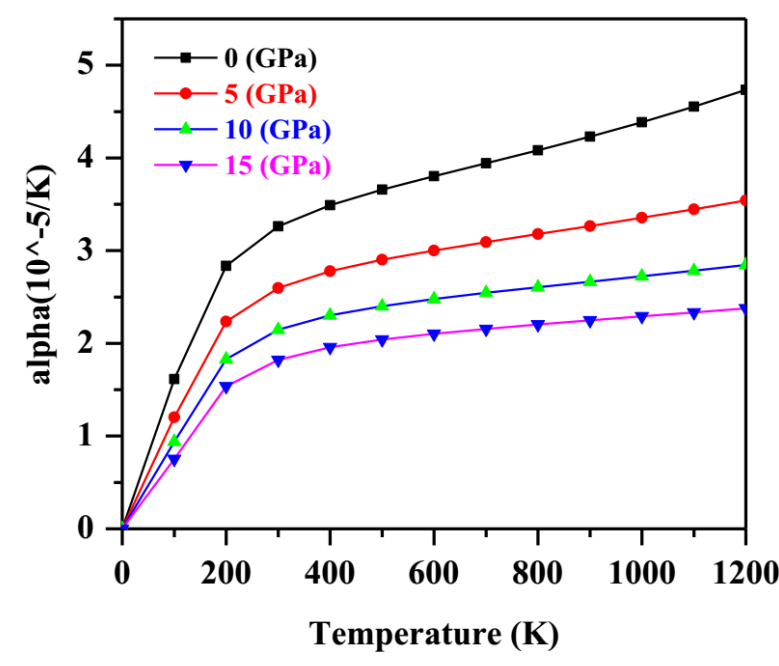

Fig. 3. Variation of the bulk modulus as a function of temperature for $\mathrm{SrThO}_{3}$ at pressure of 0, 5, 10 and $15 \mathrm{GPa}$ for $\mathrm{SrThO}_{3}$.

increase of temperature up to $200 \mathrm{~K}$. When $T>200 \mathrm{~K}, \alpha$ gradually approaches a linear increase, which means that the temperature dependence of $\alpha$ is very small at high temperature. At $400 \mathrm{~K}$ and zero pressure, $\alpha$ is equal to $3.490 \times 10^{-5} \mathrm{~K}^{-1}$.

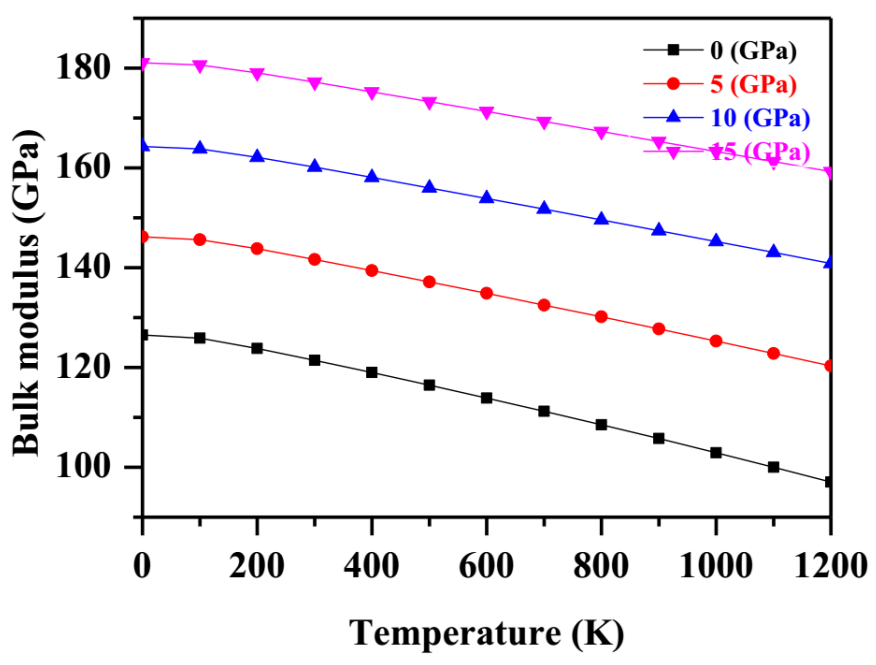

Fig. 4. Variation with temperature of the thermal expansion coefficient, at pressure of $0,5,10$ and $15 \mathrm{GPa}$ for $\mathrm{SrThO}_{3}$.

The investigation of the heat capacity of crystals is an old topic of condensed matter physics with which illustrious names are associated [37]. We show in Fig. 5 variation of the heat capacities $C_{V}$ versus temperature at 0 to $15 \mathrm{GPa}$. It is shown that when $T<600 \mathrm{~K}$, the heat capacity $C_{V}$ is depending on both the temperature and the pressure. When the temperature is constant, $C_{V}$ decreases with the applied pressure. At high temperature $(T>600 \mathrm{~K}) C_{V}$ tends to the DulongPetit limit [38], which is common to all solids at high 
temperature. At high temperature $C_{V}$ tends to approach $121.86 \mathrm{~J} \mathrm{~mol}^{-1} \mathrm{~K}^{-1}$. At zero pressure and $300 \mathrm{~K}$, $C_{V}$ is equal to $113.41 \mathrm{~J} \mathrm{~mol}^{-1} \mathrm{~K}^{-1}$.

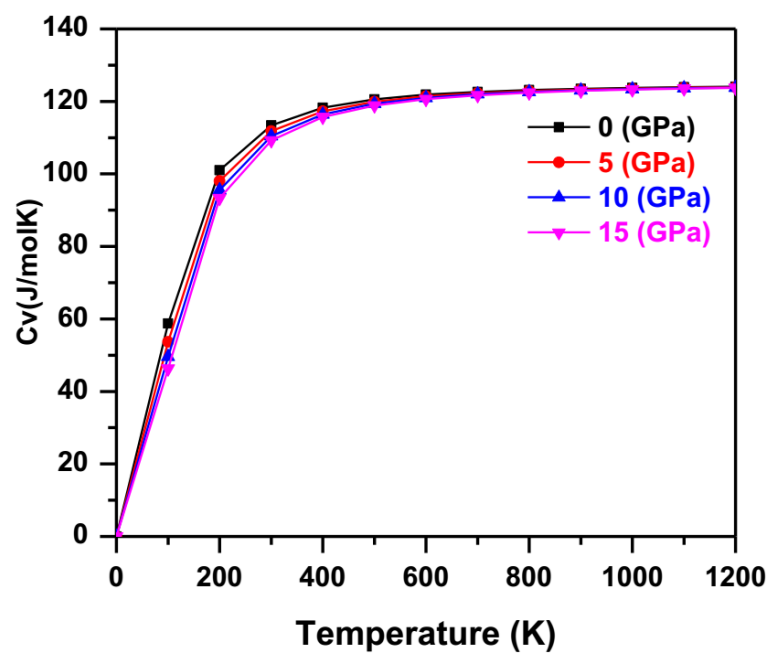

Fig. 5. Variation with temperature of the specific heat at constant volume, $C_{V}$, at pressure of $0,5,10$ and $15 \mathrm{GPa}$ for $\mathrm{SrThO}_{3}$.

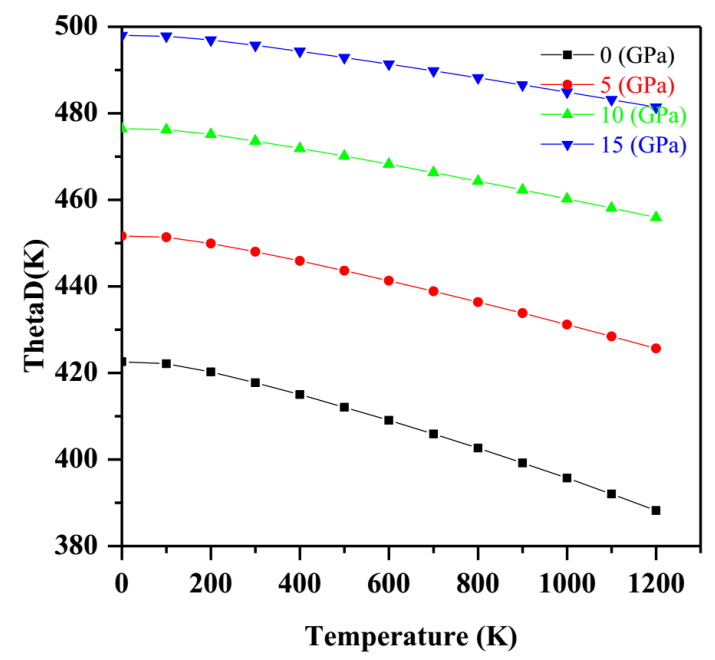

Fig. 6. Variation with temperature of the Debye temperature, $\theta_{\mathrm{D}}$ at pressure of $0,5,10$ and $15 \mathrm{GPa}$ for $\mathrm{SrThO}_{3}$.

Finally, in Fig. 6, we present the dependence of the Debye temperature $\theta_{\mathrm{D}}$ on temperature and pressure. It can be seen that $\theta_{\mathrm{D}}$ is nearly constant from 0 to $100 \mathrm{~K}$ and increases linearly with increasing temperature from $T>200 \mathrm{~K}$. It is also shown that when the temperature is constant, the Debye temperature increases almost linearly with applied pressure. Our calculated $\theta_{\mathrm{D}}$ at zero pressure and zero temperature is equal to $422.58 \mathrm{~K}$, which is in agreement with the value of $324 \mathrm{~K}$ computed accurately in terms of the elastic constants (Table II). This might be an indication that the quasi-harmonic Debye model is a very reasonable alternative to account for the thermal effects, with no expensive task in terms of computational time.

\subsection{Electronic properties}

The band structure, charge density and the electronic density of states are calculated with the well converged self-consistent solution of FP-LAPW, shown in Fig. 3. The band structure is calculated along the high symmetry directions of the 1st Brillouin zone, using two approximations, LDA and GGA. The zero of energy is chosen to coincide with the Fermi energy level. It is clear from Fig. 7 that, $\mathrm{SrThO}_{3}$ is a direct band gap compound at the high point of symmetry $\Gamma$ with a band gap of $4.23 \mathrm{eV}$, calculated with $\mathrm{mBj}$ method. In this compound, the upper valence band maximum and the lower conduction band minimum occur at $\Gamma$ points of the Brillouin zone (the optical transitions are direct). The important features of the band structure for this compound are given in Table I along with the results of previous works.
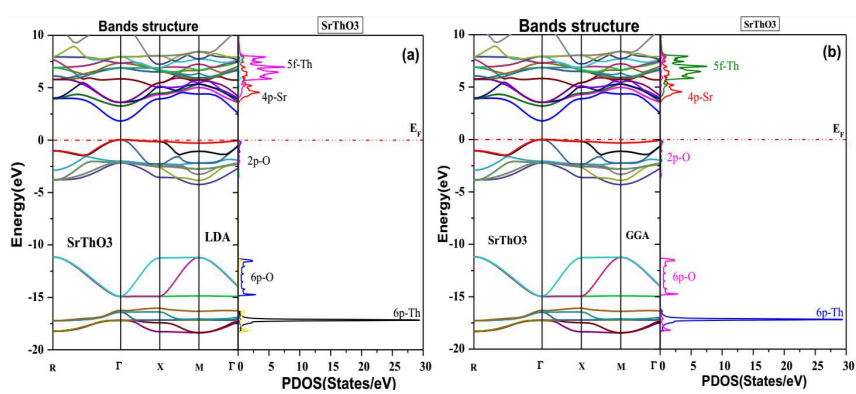

Fig. 7. Energy band structure and partial density of states (PDOS) of $\mathrm{SrThO}_{3}$ (a) LDA approximation and (b) GGA approximation .

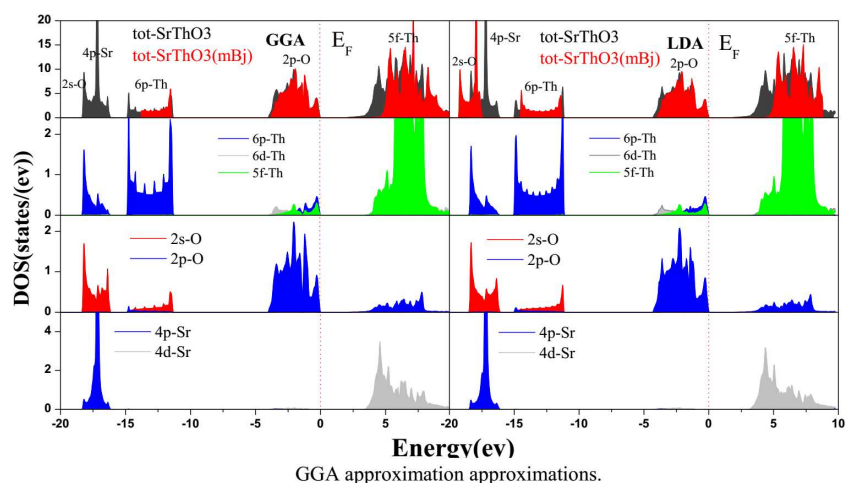

Fig. 8. Total and partial density of states for $\mathrm{SrThO}_{3}$ within LDA, GGA and mBJ approximations.

The calculated total (TDOS) and partial density of states for $\mathrm{SrThO}_{3}$ is shown in Fig. 8. The overall TDOS profiles are in good agreement with previous theoretical results [10]. It is clear from the figures that the density of states can be mainly divided into four parts. The first and second part are located between -18.30 and $-16 \mathrm{eV}$ and between -14.80 and $-11.30 \mathrm{eV}$, respectively. The first and the second region are dominated with O-2s, Sr- $4 \mathrm{p}$ and Th-6p respectively; the third part from -3.90 to $0 \mathrm{eV}$ in the valence band is mainly made from of $\mathrm{O}-2 \mathrm{p}$ orbitals; the forth part extending from 2.25 to $8 \mathrm{eV}$ in 
the conduction band. The conduction band for $\mathrm{SrThO}_{3}$ consists of the Th-5f with Th-6d character. It is clear for $\mathrm{SrThO}_{3}$, that the covalent bonds in the crystal are due to the mixing of oxygen $2 \mathrm{p}$ orbital with thorium $6 \mathrm{~d}$ and $5 \mathrm{f}$ orbitals, whereas in this energy region the occupied $\mathrm{Sr}$ states are practically absent at the Fermi level energy.

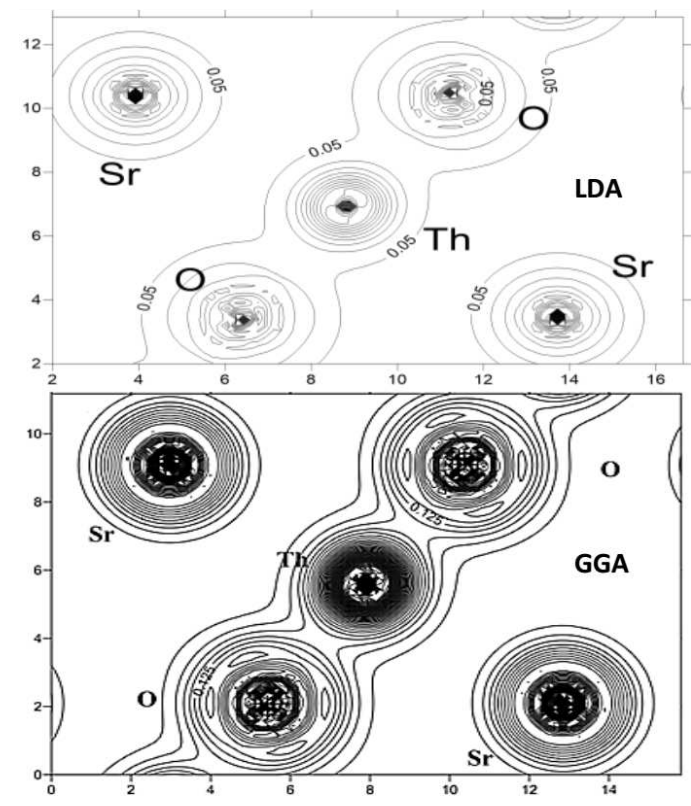

Fig. 9. Total electron density for $\mathrm{SrThO}_{3}$ in the $(110)$ plane, calculated with GGA and LDA approximation.

Charge density maps serve as a complementary tool for achieving a proper understanding of the electronic structure of the system being studied. We have calculated the charge density of $\mathrm{SrThO}_{3}$ in the (110) plane. It can be seen from Fig. 9 that most of the charge density is located in the Th-O bond direction and there is a large transfer of charge among $\mathrm{Sr}$ and $\mathrm{O}$ atoms with a very small contour among the ions, hence $\mathrm{Sr}-\mathrm{O}$ bond is strongly ionic with very weak covalent nature. We also show that electrons are strongly shared and distributed along the $\mathrm{Th}-\mathrm{O}$ bond. Hence, this bond is strongly covalent with the bond length of $2.31 \AA$.

\subsection{Optical properties}

In this theoretical method, the dielectric function and other optical properties can be calculated by using the momentum matrix elements. The optical properties of the cubic perovskite $\mathrm{SrThO}_{3}$ can be calculated using complex dielectric function $\varepsilon(\omega)=\varepsilon_{1}(\omega)+i \varepsilon_{2}(\omega)$, where $\varepsilon_{1}(\omega)$ is the real part and $\varepsilon_{2}(\omega)$ is the imaginary part of the dielectric function. It is directly related to the electronic band structure of the material and describes the absorptive behaviour.

In this section, we present in Figs. 10 and 11 the optical properties, as well as, the dielectric function, the refractive index, the absorption coefficient, and the optical conductivity of $\mathrm{SrThO}_{3}$.

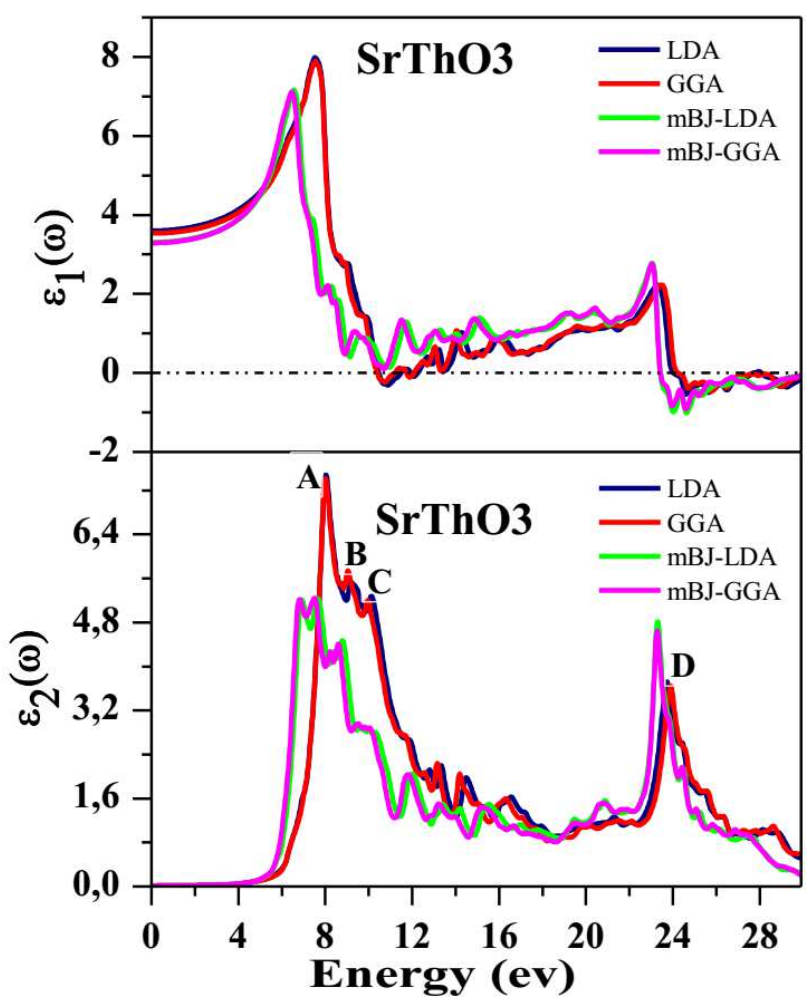

Fig. 10. Real and imaginary part of dielectric function of $\mathrm{SrThO}_{3}$.

The imaginary part of dielectric function is depicted in Fig. 10. The optical band gap obtained from this function is $4.39 \mathrm{eV}$, which is close to the values obtained from the band structure. The gap was shifted closer to the experimental data due to mBJ results.

The maxima in the imaginary part of $\varepsilon_{2}(\omega)$ are four peaks $\mathrm{A}, \mathrm{B}, \mathrm{C}$ and $\mathrm{D}$ at $7.4,5.7,5.2$ and 3.6 , corresponding to the energies of $8,9,12$ and $24 \mathrm{eV}$ respectively, in the GGA approximation. The origin of these peaks lies in the inter band transitions which can be related to the density of states of the compound shown in Fig. 8. The peaks are due to the transition of electrons from $2 \mathrm{p}-\mathrm{O}$ to $4 \mathrm{~d}-\mathrm{Sr}$ and $5 \mathrm{f}-\mathrm{Th}$ states, and from $6 \mathrm{~d}-\mathrm{Th}, 4 \mathrm{~d}-$ $\mathrm{Sr}$ to $5 \mathrm{~d}-\mathrm{Th}$ states in the conduction band. It is clear from these values that the band gap of $\mathrm{SrThO}_{3}$ is larger than $3.1 \mathrm{eV}$. Thus it should work well in the ultraviolet (UV) region of the spectrum [39, 40]. This direct and wide band gap material could be suitable for the high frequency UV device applications.

Similarly, using mBJ-LDA and mBJ-GGA we have found three high peaks $5.2,4.4$ and $2 \mathrm{eV}$, corresponding to the energies of $6.8,7.5,8.5$ and $12 \mathrm{eV}$. The peaks are from the interband transitions between the $\mathrm{O}-2 \mathrm{p}$ to Th-5d.

The high peaks in the vacuum ultraviolet energy regions indicate the strong optical activity of this material, which can be used for optoelectronic devices.

The real part of dielectric function $\varepsilon_{1}(\omega)$ is displayed in Fig. 10. The calculated static dielectric constant $\varepsilon_{1}(0)$ 


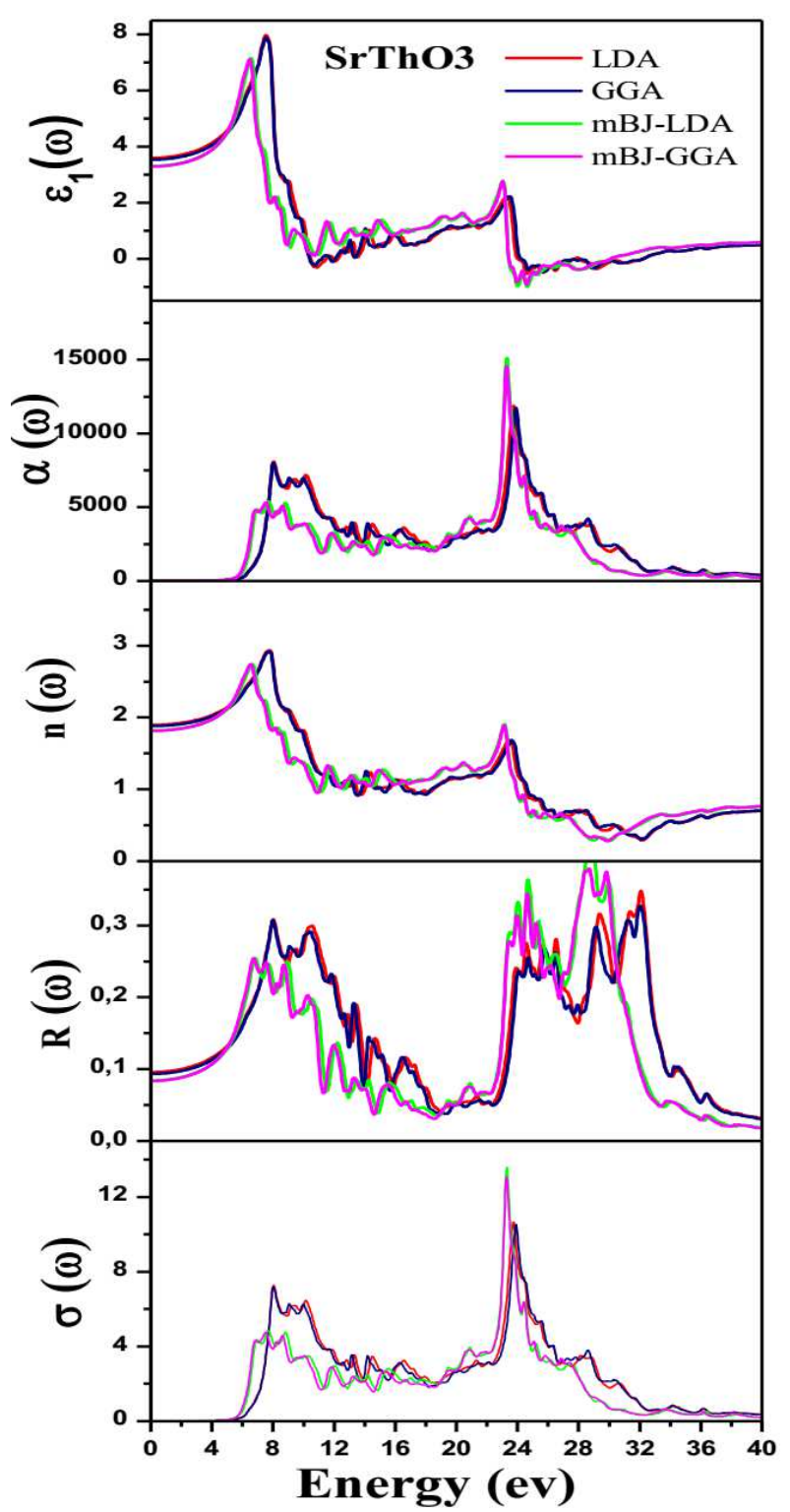

Fig. 11. Optical parameters of $\mathrm{SrThO}_{3}$.

is at about $3.52 \mathrm{eV}$ within GGA and $2.86 \mathrm{eV}$ within LDA approximation. The static dielectric constant is very important in GGA compared to LDA. The main peak is at about 7.10 within LDA and 7.55 within GGA at $7.63 \mathrm{eV}$ and $7.88 \mathrm{eV}$, respectively. Above the maximum the curve decreases and becomes flattened with small variations. It is further noted that the real part of dielectric function become negative in the energy ranges of $10.40-11.19 \mathrm{eV}$, $11.74-12.06 \mathrm{eV}$ and $24.25-32.20 \mathrm{eV}$. In these ranges the photon beam is completely absorbed in the optical medium and the material shows metallic nature.

Figure 11 shows a strong absorption nature in the different parts of spectra, ranges $3.30-30 \mathrm{eV}$. Thus this material can be also used as a filter for various energies in the UV spectrum.

The knowledge of $\varepsilon_{1}(\omega)$ and $\varepsilon_{2}(\omega)$ allows using equations

$$
\begin{aligned}
& \alpha(\omega)=\frac{2 \pi}{\lambda} K(\omega), \\
& \alpha(\omega)=\frac{2 \pi \omega}{c} \sqrt{\frac{-R e(\varepsilon(\omega)|\varepsilon(\omega)|)}{2},} \\
& n(\omega)=\frac{1}{2}\left[\varepsilon_{1}(\omega)^{2}+\left(\varepsilon_{2}(\omega)^{2}\right)^{\frac{1}{2}}+\varepsilon_{1}(\omega)\right]^{\frac{1}{2}}, \\
& R=\left|\frac{N-1}{N+1}\right|=\frac{(n-1)^{2}+K}{(n+1)^{2}+K}, \\
& \sigma(\omega)=-\frac{i \omega}{4 \pi} \varepsilon(\omega) .
\end{aligned}
$$

The critical point of the absorption coefficient $\alpha(\omega)$ (Fig. 11) is at $6.21 \mathrm{eV}$. The compound has strong response to the incident photons in the range $6.25-32 \mathrm{eV}$. The highest peak value, which corresponds to the maximum absorption of $\alpha(\omega)$, is at $23 \mathrm{eV}$.

The refractive index $n(\omega)$ is displayed in Fig. 11 along XX-direction. The static refractive index $n_{0}(\omega)$ is found to be 1.68 within LDA and 1.88 within GGA approximation. The refractive index reaches a maximum value of 2.80 within LDA and 2.92 within GGA at $7.90 \mathrm{eV}$ and $7.76 \mathrm{eV}$, respectively.

The reflection coefficient is a very important parameter which characterizes the part of reflected energy at the interface of the solid. From Fig. 11, the zero frequency limit of reflectivity of $\mathrm{SrThO}_{3}$ is found to be 0.065 within LDA and 0.093 within GGA approximation. There are high reflection peaks at energies 8.5, 10.5, 13.5 and $29 \mathrm{eV}$ corresponding to the negative value of $\varepsilon_{1}(\omega)$.

The optical conductivity is a complex quantity. This complex quantity was also calculated and is shown in Fig. 11. From the figure it is noted that the optical conductance starts responding to the applied energy field from $5.79 \mathrm{eV}$. The good response is found in the range 5.8-32 eV. The maximum optical conductivity of the compounds is at about $23.74 \mathrm{eV}$.

\section{Conclusions}

We presented our results of the optoelectronic, structural, thermal and elastic properties of $\mathrm{SrThO}_{3}$ in the cubic phase, calculated using the FP-LAPW method with the LDA, GGA approximations and mBJ. By comparison with other reports we confirm that theoretically predicted lattice constant and bulk modulus of this compound are in a good agreement with the available data. The independent elastic constants and their pressure derivatives are evaluated and linear pressure dependences of the bulk modulus and elastic constants are found. Through the quasi-harmonic Debye model, the dependences of the lattice constant, bulk modulus, thermal expansion parameter, heat capacity and Debye temperature on temperature and pressure have been obtained and compared successfully. Moreover, we have noticed that there is a small difference between the LDA and GGA approximation results in the most parts of the properties study. This difference is remarkable in the density of 
state. Consequently, we have introduced the $\mathrm{mBj}$ approximation that gives a correct band gap, allowing to define the correct transition in the imaginary part of dielectric function. The direct and wide band gap with the spectra of the imaginary part of the dielectric function confirm that this material is useful for optoelectronic device applications in the UV region of spectrum.

\section{References}

[1] G. Murtaza, I. Ahmad, B. Amin, A. Afaq, M. Maqbool, J. Maqssod, I. Khan, M. Zahid, Opt. Mater. 33, 553 (2011).

[2] J.M.D. Coey, M. Viret, S.V. Molnaacuter, Adv. Phys. 48, 167 (1999).

[3] I. Mary-Szabo, Publ. Univ. Tech. Sci. Budapest 1 , 30 (1947).

[4] R. Subasri, C. Mallika, T. Mathews, V.S. Sastry, O.M. Sreedharan, J. Nucl. Mater. 312, 249 (2003).

[5] M. Ali (Basu), R. Mishra, S.R. Bharadwaj, A.S. Kerkar, S.D. Dharwadkar, D. Das, J. Nucl. $\mathrm{Ma}$ ter. 299, 165 (2001).

[6] R. Prasad, S. Dash, S.C. Parida, Z. Singh, V. Venugopal, J. Nucl. Mater. 312, 1 (2003).

[7] R.D. Purohit, A.K. Tyagi, M.D. Mathews, S. Saha J. Nucl. Mater. 280, 51 (2000).

[8] K. Kamata, T. Nakamura, T. Sata, Chem. Lett. 4, $81(1975)$

[9] PC-PDF data, JCPDS-ICDD, 1990, p. 4.

[10] I.R. Shein, K.I. Shein, A.L. Ivanovskii, J. Nucl. Mater. 361, 69 (2007).

[11] P. Blaha, K. Schwarz, G.K.H. Madsen, D. Kvasnika, J. Luitz, WIEN2k, Technical Universität Wien, Austria 2001.

[12] D.M. Ceperley, B.J. Alder, Phys. Rev. Lett. 45, 566 (1980).

[13] J.P. Perdew, A. Zunger, Phys. Rev. B 23, 5048 (1981).

[14] J.P. Perdew, K. Burke, M. Ernzerhof, Phys. Rev. Lett. 77, 3865 (1996).

[15] D. Koller, F. Tran, P. Blaha, Phys. Rev. B 83 195134 (2011).

[16] H. Dixit, R. Saniz, S. Cottenier, D. Lamoen, B. Partoens, J. Phys. Cond. Matt. 24, 205503 (2012).

[17] D. Singh Phys. Rev. B. 82, 155145 (2010).
[18] V. Milman, M.C. Warren, J. Phys. Cond. Matt. 13, 241 (2001).

[19] M.A. Blanco, E. Francisco, V. Luana, Comput. Phys. Comm. 158, 57 (2004)

[20] M.A. Blanco, E. Francisco, V. Luafia, Comput. Phys. Commun. 158, 57 (2004).

[21] R.L. Moreira, A. Dias, J. Phys. Chem. Solids 68 , 1617 (2007)

[22] A.I. Lebedev, J. Alloy Compd. 580, 487 (2013).

[23] R. Ubic, J. Am. Ceram. Soc. 90, 3326 (2007).

[24] A.S. Verma, A. Kumar, S.R. Bhardwaj, Phys. Stat. Solid. B 245, 1520 (2008).

[25] N. Xu, H. Zhao, X. Zhou, W. Wei, X. Lu, W. Ding, F. Li, J. Hydrogen Energy 35, 7295 (2010).

[26] V.M. Goldschmidt, Skrifer Norske Videnskaps-Akad. Oslo, I. Mat.-Nat. K1, 8 (1926)

[27] C. Kittel, Introduction to Solid State Physics, John Wiley \& Sons, USA 1996.

[28] S. Yip, J. Li, M. Tang, J. Wang, Mater. Sci. Eng. A 317, 236 (2001)

[29] G.V. Sin'ko, A. Smimov, J. Phys. Cond. Matt. 14, 6989 (2002)

[30] B. Mayer, H. Anton, E. Bott, M. Methfessel, J. Sticht, J. Harris, P.C. Schmidt, Intermetallics 11, 23 (2003).

[31] W.A. Harisson, Electronic Structure and the Properties of solids, Dover Publications, 1989.

[32] J. Haines, J.M. Leger, G. Bocquillon, Ann. Rev. Mater. Res. 31, 1 (2001).

[33] S.F. Pugh, Phil. Mag. 45, 833 (1954).

[34] O.L. Anderson, J. Phys. Chem. Solids 24, 909 (1963).

[35] E. Johnston, G. Keeler, R. Rollins, S. Spicklemeire, Solid State Physics Simulations, A Consortium for Upper Level Physics Software, Wiley, New York 1996.

[36] O.L. Anderson, J. Phys. Chem. Solids 24, 909 (1963).

[37] P. Debye, Annalen Der Physik 39, 789 (1912).

[38] A.T. Petit, P.L. Dulong, Annales de Chimie et de Physique 10, 395 (1819).

[39] M. Maqbool, I. Ahmad, H.H. Richardson, M.E. Kordesch, Appl. Phys. Lett. 91, 193511 (2016).

[40] B. Amin, I. Ahmad, M. Maqbool, J. Lightwave Technol. 28, 223 (2010). 